\title{
A case report of membranous occlusion of the subclavian vein: a rare cause of McCleery syndrome
}

\author{
Jingjing Yin ${ }^{1}$, Zhenhong $\mathbf{Q i}^{2}$, Yu Chen ${ }^{3}$, Yuexin Chen ${ }^{4}$ \\ ${ }^{1}$ Peking Union Medical College Hospital, Chinese Academy of Medical Sciences and Peking Union Medical College, Beijing, China; ${ }^{2}$ Department \\ of Ultrasonography, Peking Union Medical College Hospital, Chinese Academy of Medical Sciences and Peking Union Medical College, Beijing, \\ China; ${ }^{3}$ Department of Radiology, Peking Union Medical College Hospital, Chinese Academy of Medical Sciences and Peking Union Medical \\ College, Beijing, China; ${ }^{4}$ Department of Vascular Surgery, Peking Union Medical College Hospital, Chinese Academy of Medical Sciences and \\ Peking Union Medical College, Beijing, China \\ Correspondence to: Yuexin Chen, MD. Department of Vascular Surgery, Peking Union Medical College Hospital, Chinese Academy of Medical \\ Sciences and Peking Union Medical College, 1 Shuaifuyuan, Beijing 100730, China. Email: cyuexin2007@163.com.
}

\begin{abstract}
Patients presenting with periodic swelling of the upper extremity without thrombosis are diagnosed with McCleery syndrome. There have been sporadic cases reported over the past decades. Due to the rarity of this disease, no standard consensus on diagnosis and treatment of McCleery syndrome was established. Subclavius tendon and anterior scalene muscle compression were proposed as the primary cause of McCleery syndrome. Partial resecting muscle, tendon or ligament was recommended as therapies. We report one rare case of membranous occlusion of the subclavian vein (SCV) that leads to periodic swelling of upper extremity and diagnosis of McCleery syndrome was made. This 21-year-old man complained of swelling and pain in the right upper extremity after strenuous exercise lasting for 3 months. Physical examination, spinal $\mathrm{X}$-ray and magnetic resonance imaging showed no signs related to classic venous thoracic outlet syndrome (VTOS). Duplex ultrasonography demonstrated membranous occlusion without thrombosis at the proximal end of the right SCV. The lesion was confirmed by venography. Treated by balloon dilation alone, the patient recovered uneventfully during 18 months of follow-up. Repeated duplex ultrasonography revealed patency of the SCV. To our best knowledge, our case provides the first reported membranous occlusion of the SCV. Excluding the presence of thrombosis in SCV, he was diagnosed with McCleery syndrome and was cured by balloon dilation alone. We can learn from this rare case that membranous occlusion of veins can be a rare cause of McCleery syndrome and is worthy of careful consideration and differentiation of VOTS.
\end{abstract}

Keywords: Subclavian vein (SCV) occlusion; membranous lesion; thoracic outlet syndrome; case report

Submitted Mar 25, 2020. Accepted for publication Sep 30, 2020.

doi: $10.21037 /$ atm-20-2862

View this article at: http://dx.doi.org/10.21037/atm-20-2862

\section{Introduction}

McCleery syndrome is defined as intermittent obstruction of the subclavian vein (SCV) without thrombosis. It was first described by Charles W. McLaughlin Jr in 1939 and further explained in detail by McCleery and colleagues in $1951(1,2)$. Traditionally, the cause of McCleery syndrome is believed to be venous thoracic outlet syndrome (VTOS). Patients with VTOS usually have pain, numbness, paresthesia or weakness in the upper limbs due to compression of SCV. There have been many causes of VTOS, including arterial thrombosis, neurological compression or PagetShroette "effort" thrombosis $(3,4)$. Patients with VTOS may undergo first rib resection and scalenectomy (FRRS) to relieve compression. Balloon angioplasty would be done if postoperative venogram reveals stenosis of the SCVs. McCleery syndrome is a distinct disease entity presenting as VTOS, but with no thrombosis. The primary cause 
was proposed to be subclavius tendon and anterior scalene muscle compression. Recommended therapies are to partially resect the subclavius muscle and tendon, partially excising the costocoracoid ligament or dividing the anterior scalene attachment to the first rib (2). We report a rare case of McCleery syndrome caused by membranous occlusion. Membranous occlusion of veins is rare and mostly reported in the suprahepatic inferior vena cava (IVC), resulting in hepatic venous outflow obstruction. Angioplasty by balloon dilations can be a safe and effective treatment for membranous occlusion of IVC (5-8). Our patient was also relieved by balloon dilation of SCV and recovered successfully. We present the following case in accordance with the CARE reporting checklist (available at http:// dx.doi.org/10.21037/atm-20-2862).

\section{Case presentation}

A 21-year-old man complained of swelling, edema and severe pain in the right upper extremity after strenuous exercise lasting for three months. The symptoms exacerbated when lifting heavy objects and relieved after rest. He denied any numbness, dysesthesia or weakness occurring in the right hand and arm. He reported no history of trauma, malignancy, family history of thrombosis, or a history of treatment with central venous catheters or pacemakers. He was admitted to our hospital for further diagnosis and treatment. Physical examination showed arm swelling with bluish cyanosis. Dilated subcutaneous veins were present over the affected arm. The radial pulse was regular and the Adson and Elvey tests were negative.

Spinal X-ray was normal without signs of skeletal abnormalities, such as cervical ribs, clavicular anomalies and isolated first rib abnormalities. Duplex ultrasonography demonstrated a fornix-shaped membrane located at the proximal end of the right SCV, and the venous flow through the membrane was narrowed with a width of $0.14 \mathrm{~cm}$ (Figure 1). When executing the provocative maneuvers (moving the patient's upper extremity into an extended, abducted and externally rotated position), the width of venous flow through the membrane gently enlarged to $0.37 \mathrm{~cm}$. There was no sign of thrombosis in the SCV. Magnetic resonance imaging (MRI) was also applied, but no anatomical abnormalities of soft tissue or signs of vascular compression were identified (Figure 2). Complete blood count, D-dimer, protein C, protein S, antithrombin III and homocysteine tests were normal.

Low-molecular-weight heparin $(6,000 \mathrm{U}$ once per day) was administered to this patient to prevent secondary thrombosis in the SCV. Subsequently, digital subtraction venography was considered for this patient. He was scheduled in a supine position with the right arm at 90 degrees. After the administration of local anesthesia, a 6-F sheath was inserted into the cephalic vein at the right elbow. After the infusion of 6,000 U heparin, we used a 5-F vertebral catheter and a 5-F pigtail catheter for venography, which indicated a fornix-shaped membrane at the proximal segment of the SCV without signs of thrombosis. The blood flow was almost completely obstructed by the membrane (Figure 3). There were no obvious collaterals around the occlusion.

Moving the arm from full adduction to full abduction resulted in no obvious changes in the degree of occlusion or the shape of the SCV. A 0.035 -inch Terumo guide wire was gently slipped through the slim hole of the membrane. The lesion was gradually dilated using an $8-40 \mathrm{~mm}$ balloon (Mustang $^{\mathrm{TM}}$, Boston Scientific Co., Ltd., USA) and a 12-40 mm balloon (Mustang ${ }^{\mathrm{TM}}$, Boston Scientific Co., Ltd., USA). The patient suffered from severe pain in the subclavian area during inflation, and the pain disappeared after deflation. Finally, the blood flow of the SCV was restored, with residual stenosis of less than $30 \%$. Six months of anticoagulation treatment with $20 \mathrm{mg} / \mathrm{d}$ rivaroxaban was administered to prevent recurrent thrombosis in the SCV.

During follow-up, the patient recovered without any complication. The swelling and pain of his right upper limb disappeared. Twelve months after the surgery, repeated duplex ultrasonography revealed patency of the SCV without apparent abnormalities (Figure 4). And he remained unsymptomatic at the time of 18 months of follow-up.

All procedures performed in studies involving human participants were in accordance with the ethical standards of the institutional and/or national research committee(s) and with the Helsinki Declaration (as revised in 2013). Written informed consent was obtained from the patient.

\section{Discussion}

Intermittent obstruction of the SCV without thrombosis was described by McCleery and colleagues and was called McCleery syndrome (1). Mostly, the pathology results from the compression of the vein between the subclavius tendon and the anterior scalene muscle that is worsened with arm abduction. In this case, we found a different cause of McCleery syndrome from VTOS. In our young man, the SCV was obstructed by a membranous structure without 

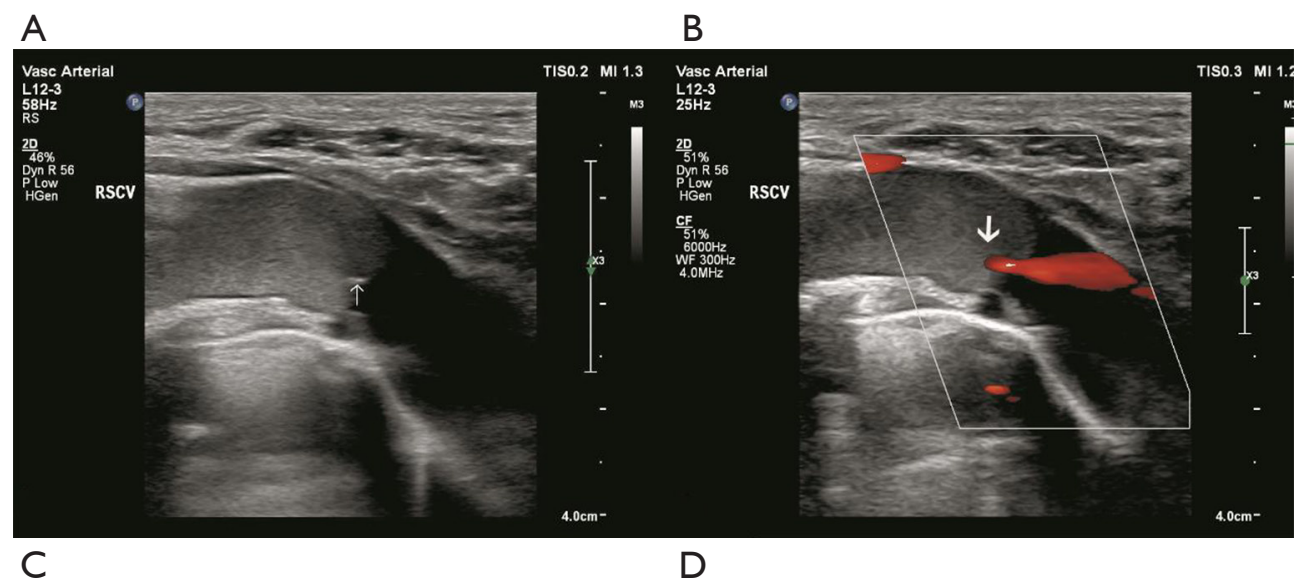

D

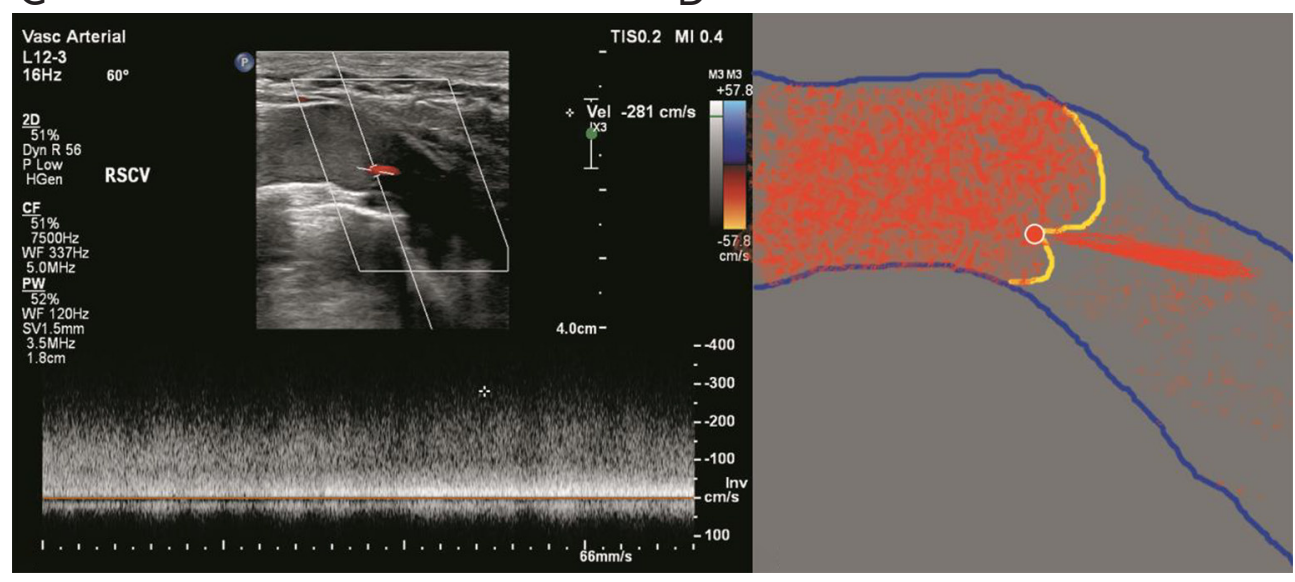

Figure 1 Duplex ultrasonography showed the location of the membrane occlusion of the SCV. (A) Duplex ultrasonography demonstrated a fornix-shaped membrane (white arrow) located at the proximal end of the right SCV. The blood flow was slow distal to the membrane. (B) Color Doppler ultrasound showed the blood flow being forced out from the small orifice (white arrow) of the membrane. (C) The maximal velocity of blood flow at the orifice was up to $281 \mathrm{~cm} / \mathrm{s}$. (D) Sketch of the SVC, fornix-shaped membrane and orifice.

venous compression or thrombosis. It is the first reported case of McCleery syndrome caused by membranous occlusion of the SCV.

However, the current understanding of membranous occlusion of veins mainly comes from obstruction of IVC. Membranous obstruction of the suprahepatic IVC is a rare cause of Budd-Chiari syndrome (BCS), which is designated as an obstructed IVC segment length of $\leq 1 \mathrm{~cm}$ (9). It results from fibrous thickening of the intima and is commonly located at the orifices of the hepatic veins (10). Embryological origin and thrombotic origin have been considered to be the two possible etiopathogenic hypotheses. Congenital obstruction is an anomaly attributed to vascular malformation during fetal development (11). Other aspects, such as anatomical and infectious factors, have also been suspected to be related to the physiopathological mechanism of membranous obstruction (12). In the present case, the patient had no history of trauma, catheterization, or malignancy or family history of thrombophilia. The Adson and Elvey tests were negative. When executing the provocative maneuvers, both venography and duplex ultrasonography failed to reveal any signs of compression. We suspect the predisposing role of embryological factors in this patient, but the exact origin of the membranous structure in the SCV is still unknown.

Concerning the treatment, many studies have shown that angioplasty has excellent immediate and late efficacy in treating membranous obstruction of the IVC (MOVC) (5-8). A few surgical techniques also exist, but they are less exploited because of the superiority of endovascular treatment for its noninvasive nature and lower morbidity- 

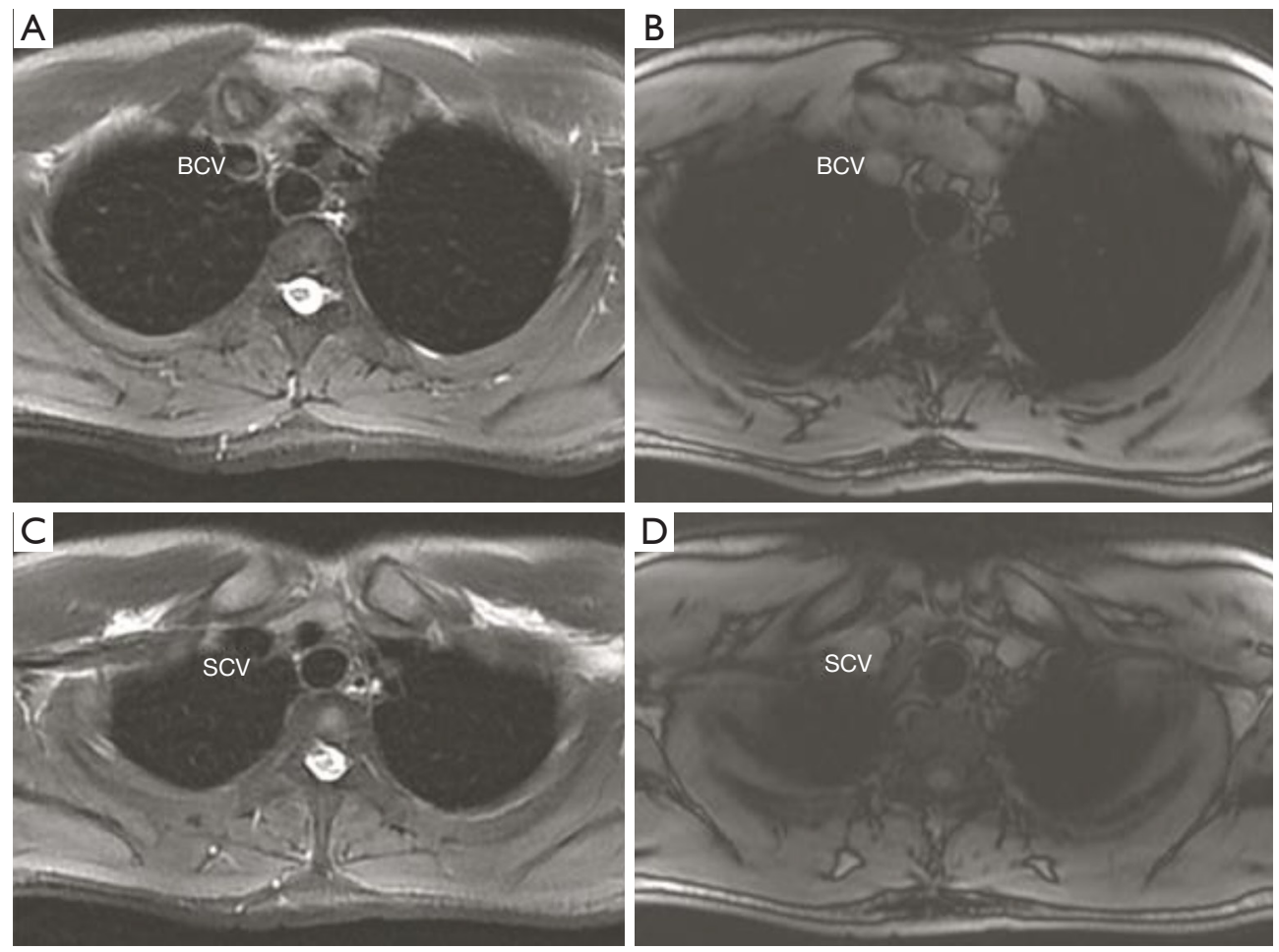

Figure 2 Magnetic resonance imaging revealed no anatomical abnormalities of soft tissue or signs of vascular compression. BCV, brachiocephalic vein; SCV, subclavian vein.

mortality rates. Balloon dilation alone could be the optimal treatment for patients with MOVC (6). Although there is no previous experience with the treatment of membranous obstruction of the SCV, we used balloon dilation alone to treat this short lesion. Without severe remnant stenosis after dilation, a self-expanding or balloon-expandable stent was not considered in this case due to the subsequent risk of complications, such as stent deformation, fracture and thrombosis, in this setting. The results at 18 months of follow-up were good; however, the long-term observation is still required. Membranous occlusion of the SCV can be confused with thrombosis. If thrombosis coexists, catheter-directed thrombolysis (CDT) and percutaneous mechanic thrombectomy (PMT) are useful for revealing the lesion underlying the thrombosis. Surgical procedures were not suitable for this patient because anatomical abnormalities were not confirmed. However, for patients with VTOS with persistent stenosis or occlusion after thrombolysis, early surgical decompression of the thoracic outlet within two weeks after CDT or PMT should be considered (13).

Systemic anticoagulation treatment is useful for preventing secondary thrombosis related to stenosis or occlusion of the SCV. After endovascular treatment, anticoagulation therapy should continue for at least 36 months to prevent recurrent thrombosis or occlusion. Our patient was administered direct oral anticoagulant therapy for six months. The patency of the SVC remains good.

Our report inevitably has limitations. In order to ensure the safety, we chose endovascular treatment instead of open surgery, where the direct view and pathological results of the membranous structure are sacrificed. And due to the extreme rarity of membranous occlusion of SVC, it is difficult to explain its exact origin.

Learning points are that membranous obstruction of the SVC could happen and caused the McCleery syndrome. Balloon dilation could be the optimal treatment. This suggests that a nonuniform origin of short segmental occlusion of the SVC. This rare cause of McCleery syndrome is worthy of careful differentiation. 

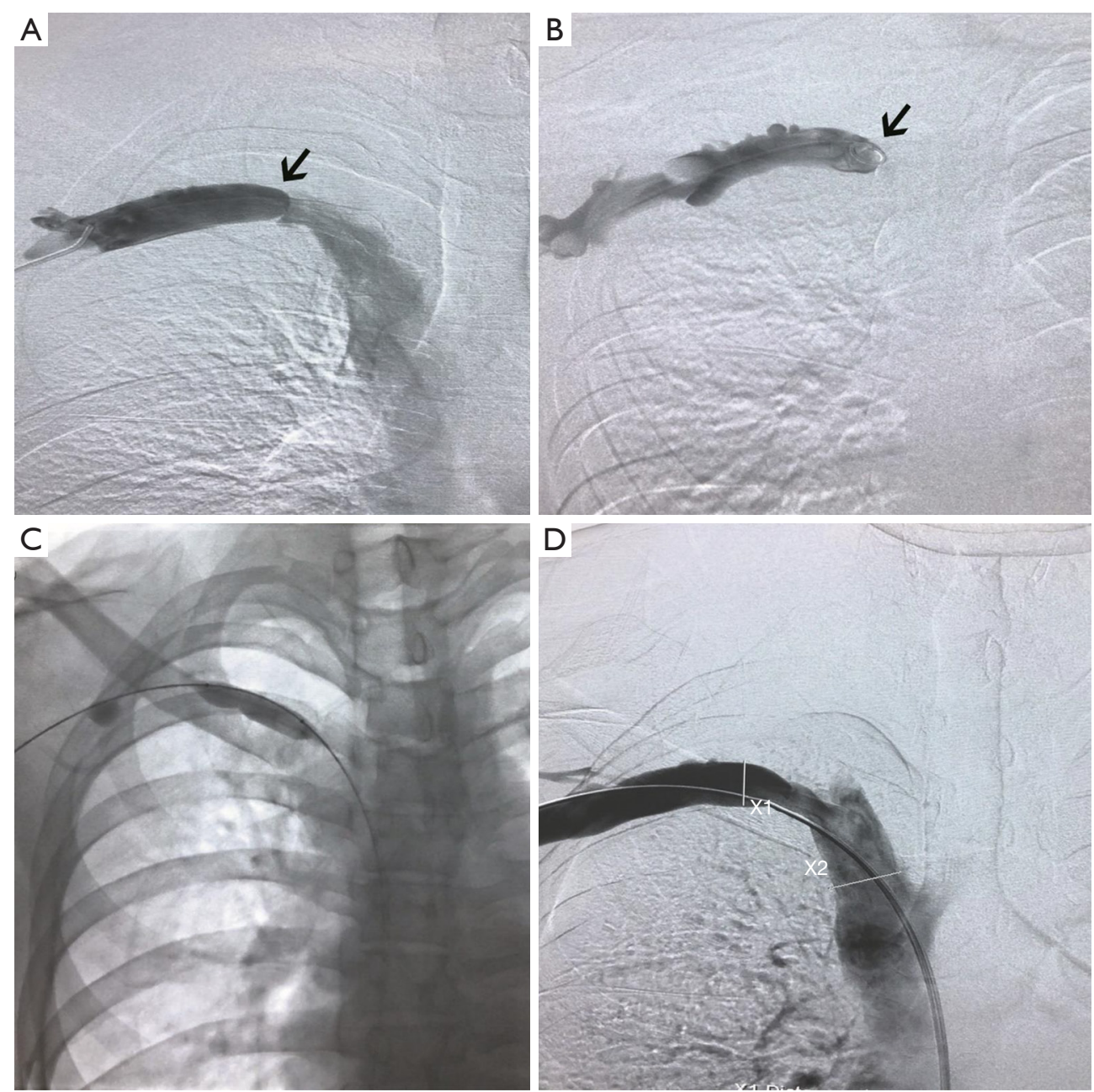

Figure 3 Venography of the SCV indicated the blood flow before and after the dilation balloon. (A,B) Venography indicated a fornix-shaped membrane (black arrow) at the proximal segment of the SCV without signs of thrombosis. The blood flow was almost completely obstructed by the membrane. There were no obvious collaterals around the occlusion. (C) The membrane was gradually dilated using an $8-40$ mm balloon and a 12-40 mm balloon. (D) After dilation, the blood flow in the SCV was recovered with residual stenosis of less than $30 \%$. SCV, subclavian vein.
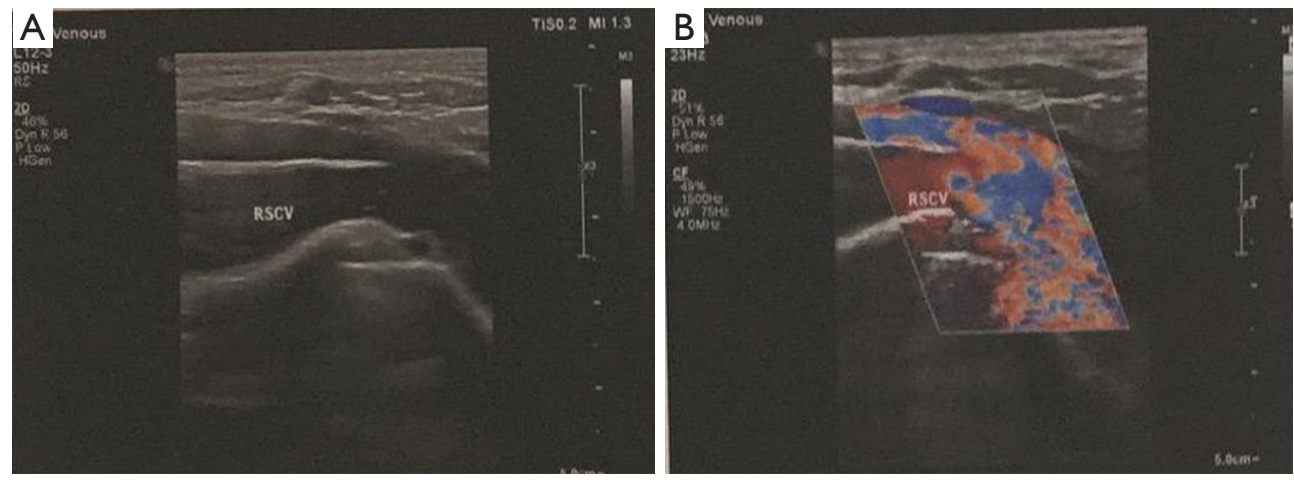

Figure 4 Duplex ultrasonography at 12 months revealed patency of the SCV without obvious abnormalities. SCV, subclavian vein. 


\section{Acknowledgments}

Funding: Our work belongs to the project of "The effect of multi-target microbubble thrombolysis on acute venous thrombosis by ultrasonography" (2019XK320020) and is supported by Basic Science Founding of Chinese Academy of Medical Sciences (NWB20206532).

\section{Footnotes}

Reporting Checklist: The authors have completed the CARE reporting checklist. Available at http://dx.doi.org/10.21037/ atm-20-2862

Peer Review File: Available at http://dx.doi.org/10.21037/ atm-20-2862

Conflicts of Interest: All authors have completed the ICMJE uniform disclosure form (available at http://dx.doi. org/10.21037/atm-20-2862). The authors have no conflicts of interest to declare.

Ethical Statement: The authors are accountable for all aspects of the work in ensuring that questions related to the accuracy or integrity of any part of the work are appropriately investigated and resolved. All procedures performed in studies involving human participants were in accordance with the ethical standards of the institutional and/or national research committee(s) and with the Helsinki Declaration (as revised in 2013). Written informed consent was obtained from the patient.

Open Access Statement: This is an Open Access article distributed in accordance with the Creative Commons Attribution-NonCommercial-NoDerivs 4.0 International License (CC BY-NC-ND 4.0), which permits the noncommercial replication and distribution of the article with the strict proviso that no changes or edits are made and the original work is properly cited (including links to both the formal publication through the relevant DOI and the license). See: https://creativecommons.org/licenses/by-nc-nd/4.0/.

\section{References}

1. McCleery RS KJ, Kirtley JA, Love RB. Subclavius and anterior scalene muscle compression as a cause of intermittent obstruction of the subclavian vein. Ann Surg 1951;133:588-602.

2. Likes K, Rochlin D, Call D, et al. McCleery syndrome: etiology and outcome. Vasc Endovascular Surg 2014;48:106-10.

3. Chang K, Likes K, Demos J, et al. Routine venography following transaxillary first rib resection and scalenectomy (FRRS) for chronic subclavian vein thrombosis ensures excellent outcomes and vein patency. Vasc Endovascular Surg 2012;46:15-20.

4. Swinton N, Edgett J, Hall R. Primary subclavian-axillary vein thrombosis. Circulation 1968;38:737-45.

5. Kucukay F, Akdogan M, Bostanci EB, et al. Percutaneous Transluminal Angioplasty for Complete Membranous Obstruction of Suprahepatic Inferior Vena Cava: Long-Term Results. Cardiovasc Intervent Radiol 2016;39:1392-9.

6. Huang Q, Shen B, Zhang Q, et al. Comparison of Long-Term Outcomes of Endovascular Management for Membranous and Segmental Inferior Vena Cava Obstruction in Patients With Primary Budd-Chiari Syndrome. Circ Cardiovasc Interv 2016;9:e003104.

7. Meng X, Lv Y, Zhang B, et al. Endovascular Management of Budd-Chiari Syndrome with Inferior Vena Cava Thrombosis: A 14-Year Single-Center Retrospective Report of 55 Patients. J Vasc Interv Radiol 2016;27:1592-603.

8. Zhang W, Wang QZ, Chen XW, et al. Budd-Chiari syndrome in China: A 30-year retrospective study on survival from a single center. World J Gastroenterol 2018;24:1134-43.

9. Okuda K. Membranous obstruction of the inferior vena cava (obliterative hepatocavopathy, Okuda). J Gastroenterol Hepatol 2001;16:1179-83.

10. Collot J, Bletard N, Lamproye A. Membranous occlusion of the inferior vena cava a rare cause of Budd-Chiari syndrome. Rev Med Liege 2018;73:557-61. 
11. Suah A, Millis JM, Bodzin AS. Congenital membranous occlusion of the suprahepatic inferior vena cava in a pediatric liver transplant. Hepatobiliary Surg Nutr 2018;7:29-31.

12. Shin N, Kim YH, Xu H, et al. Redefining BuddChiari syndrome: A systematic review. World J Hepatol

Cite this article as: Yin J, Qi Z, Chen Y, Chen Y. A case report of membranous occlusion of the subclavian vein: a rare cause of McCleery syndrome. Ann Transl Med 2021;9(1):78. doi: 10.21037/atm-20-2862
2016;8:691-702.

13. de Kleijn R, Schropp L, Westerink J, et al. Timing of thoracic outlet decompression following thrombolysis for primary upper extremity deep venous thrombosis. A systematic review. Ann Vasc Surg 2020;66:654-61. 